\title{
Molecular Clouds as a Probe of Cosmic-Ray Acceleration in a Supernova Remnant
}

\author{
Yutaka Fujita, Yutaka Ohira, Shuta J. Tanaka, and Fumio Takahara \\ Department of Earth and Space Science, Graduate School of Science, Osaka University, 1-1 \\ Machikaneyama-cho, Toyonaka, Osaka 560-0043, Japan
}

\begin{abstract}
We study cosmic-ray acceleration in a supernova remnant (SNR) and the escape from it. We model nonthermal particle and photon spectra for the hidden SNR in the open cluster Westerlund 2, and the old-age mixed-morphology SNR W 28. We assume that the SNR shock propagates in a low-density cavity, which is created and heated through the activities of the progenitor stars and/or previous supernova explosions. We indicate that the diffusion coefficient for cosmic-rays around the SNRs is less than $\sim 1 \%$ of that away from them. We compare our predictions with the gamma-ray spectra of molecular clouds illuminated by the cosmic-rays (Fermi and H.E.S.S.). We found that the spectral indices of the particles are $\sim 2.3$. This may be because the particles were accelerated at the end of the Sedov phase, and because energy dependent escape and propagation of particles did not much affect the spectrum.

Subject headings: radiation mechanisms: non-thermal — ISM: individual (W 28) - cosmic rays — supernova remnants — open clusters and associations: individual (Westerlund 2)
\end{abstract}

\section{Introduction}

Supernova remnants (SNRs) are canonically considered the main sources of cosmic-rays in the Galaxy. The detection of non-thermal X-ray emission from SNRs clearly indicates that electrons are actually accelerated around the SNR shocks (Koyama et al. 1995), and the observations can constrain the electron spectra. On the other hand, observational confirmation of accelerated protons is not as easy as that of electrons. One way to study the acceleration and spectrum of protons is to study gamma-ray emission through $p p$-interactions and the decay of neutral pions (e.g. Naito \& Takahara 1994; Drurv et al. 1994; Sturner et al. 1997; Gaisser, Protheroe \& Stanev 1998; Baring et al. 1999; Berezhko \& Völk 2000). In particular, molecular clouds are efficient targets of cosmic-ray protons because of their high density. 
Thus, clouds illuminated by the protons accelerated in a nearby SNR could be bright gammaray sources (e.g. Aharonian \& Atovan 1996; Fatuzzo \& Melia 2005; Fatuzzo, Adams \& Melia 2006; Gabici, Aharonian \& Casanova 2009).

Theoretical studies have suggested that old SNRs could be appropriate objects to investigate gamma-ray emission through $p p$-interactions, because the radiation from the accelerated electrons (primary electrons) disappears as the SNR evolves, owing to their short cooling time (Yamazaki et al. 2006; Fang \& Zhang 2008). In other words, we could ignore the gamma-rays from primary electrons via inverse-Compton (IC) scattering of ambient soft photon fields and/or non-thermal bremsstrahlung from the interaction of electrons with dense ambient matter.

In this letter, we consider the evolution of an SNR surrounded by molecular clouds. We calculate the spectrum of cosmic-rays accelerated in the SNR and the photon spectrum of the molecular clouds illuminated by the cosmic-rays. We assume that a supernova explodes in a low-density cavity, because the progenitor star expels ambient dense gas via strong UV-radiation and stellar winds (Chevalier 1999). The cavity may also have been created through previous supernova explosions. What differentiates this study is that we consider whether high-energy cosmic-rays illuminating molecular clouds were accelerated even after the SNR became old or they were accelerated only when the SNR was young. We also discuss the influence of particle diffusion on the cosmic-ray spectrum. We construct specific models for the open cluster Westerlund 2 and the SNR W 28, and compere the results with latest observations.

Westerlund 2 is one of the young open clusters from which $\mathrm{TeV}$ gamma-ray emission has been detected with H.E.S.S. (Aharonian et al. 2007). It is surrounded by molecular clouds (Furukawa et al. 2009). The gamma-ray emission is extended $\left(\sim 0.2^{\circ}\right)$ and covers the molecular clouds (Fukui et al. 2009). Noticeable objects such as pulsars that can be the source of the gamma-ray emission have not been observed in this region. Fujita et al. (2009) proposed that the gamma-ray emission comes from an old SNR, although there is no clear signature of SNRs in the cluster. W 28 is a mixed-morphology SNR interacting with a

molecular cloud (Wootten 1981). It is an old SNR and TeV gamma-rays have been detected from molecular clouds around the SNR (Aharonian et al. 2008).

\section{Diffusion of Cosmic-Ray Particles}

As will be shown in the next section, the proton spectrum around Westerlund 2 and W 28 can be fitted with a power-law with an index $s \sim 2.3$ (the definition is shown in 
equation (4). Moreover, the shock waves seem to have finished accelerating particles for those objects, while the surrounding regions are bright in the gamma-ray band. Here, we briefly discuss what they mean before we explain assumptions in our specific models for Westerlund 2 and W 28.

\subsection{Energy Spectrum of Cosmic-Rays}

If the duration of particle acceleration is shorter than that of the diffusion, and the particle source is spatially localized well, we can use the analytical solution in Atovan. Aharonian, \& Völk (1995). This corresponds to the situation where particles are accelerated mainly when the SNR is young and compact, and the molecular cloud illuminated by accelerated cosmic-rays is distant from the SNR shock. If the shape of the source spectrum is a power-law with an index $\alpha$ or $Q \propto E^{-\alpha} \delta(r) \delta(t)$, the energy spectrum at the position of the cloud $(r)$ is represented by

$$
f(E) \propto \frac{E^{-\alpha}}{r_{\text {diff }}^{3}} \exp \left(-\frac{r^{2}}{r_{\text {diff }}^{2}}\right),
$$

if radiative cooling during the diffusion can be ignored. The diffusion length is $r_{\text {diff }}=$

$2 \sqrt{D(E) t}$, where $D(E)$ is the diffusion coefficient. Following Gabici et al. (2009), we assume that

$$
D(E)=10^{28} \chi\left(\frac{E}{10 \mathrm{GeV}}\right)^{0.5}\left(\frac{B}{3 \mu \mathrm{G}}\right)^{-0.5} \mathrm{~cm}^{2} \mathrm{~s}^{-1},
$$

where $B$ is the magnetic field. At a point distant from an SNR, we expect that $\chi \sim 1$. Thus, for a given magnetic field, the energy spectrum is represented by $f \propto E^{-\alpha-0.75}$ if $r \lesssim r_{\text {diff }}$ at the position of the molecular cloud. This means that even if particles are accelerated efficiently $(\alpha=2)$, the energy spectrum must be soft $\left(f \propto E^{-2.75}\right)$. In other words, if the index of the spectrum is observed to be $s<2.75$ at a molecular cloud, it is likely that the particles are accelerated near the cloud after the SNR becomes large.

For Westerlund 2 and W 28, since the spectral indices are $s \sim 2.3$ for the high-energy protons $(\gtrsim \mathrm{TeV})$ that are illuminating the molecular clouds around these objects, we expect that the cosmic-rays were accelerated near the molecular clouds even after the SNRs became old and large. This may be in contrast with the assumption often adopted in theoretical studies. We assume that the SNR shock had traveled in a low-density cavity. During the propagation in the cavity, the shock wave is in the adiabatic Sedov phase, because the lowdensity prevents the radiative cooling. Thus, even if particles can be accelerated only during the Sedov phase, they are being accelerated until the shock reaches and collides with the surrounding high-density region, which is an effective target of the cosmic-rays. The particles 
illuminate the high-density region with the energy spectrum at the acceleration site or the shock. Thus, the spectral indices of $s<2.75$ are possible.

\subsection{Diffusion Coefficient}

For Westerlund 2 and W 28, efficient particle acceleration around shock waves seems to have finished. For the former, there is no signature of a shock. Thus, the shock may have already collided with the surrounding dense region and dissipated. For the latter, the shock is traveling in a relatively high density region $\left(\sim 10-100 \mathrm{~cm}^{-3}\right)$ and seems to be in a radiative phase (Rho \& Borkowski 2002). Thus, cosmic-rays that are illuminating molecular clouds might be accelerated in the past. For $\chi=1$ and $B=20 \mu \mathrm{G}$ in equation (2), Gabici et al. (2009) estimated the diffusion time of cosmic-rays in a typical molecular cloud and showed that it is only $\sim 100 \mathrm{yr}$ for protons with an energy of $\sim 10 \mathrm{TeV}$. However, the probability that the particle acceleration has finished within $\sim 100 \mathrm{yr}$ must be very small. Therefore, in the next section we simply assume that $\chi \ll 1$ around the SNRs and later discuss it quantitatively. The small $\chi$ may be because of the generation of plasma waves by cosmicrays (Wentzel 1974).

Note that if particles are accelerated with a spectral index of $\alpha=2$, and if they diffuse in the interstellar gas and illuminate molecular clouds before the index increases to $s=2.75$, the observed spectral index could be $s \sim 2.3$ as the ones observed in Westerlund 2 and W 28. However, the value of $\chi$ and/or the distance between the SNR and the molecular clouds must be fine-tuned in this case. In particular, for $\mathrm{W} 28$, the molecular clouds are adjacent to the SNR (Aharonian et al. 2008) and there seems to be no room to adjust the distance. Thus, we do not consider this possibility.

\section{Models and Results}

\subsection{Westerlund 2}

We assume that the distance to Westerlund 2 is $d=5.4 \mathrm{kpc}($ Furukawa et al. 2009). We also assume that a supernova exploded at the center of a cavity, which is filled with warm gas, and is surrounded by molecular clouds (Fig. 1a). After the explosion, a shock expands in the warm gas and then hits the surrounding molecular gas $(\sim 20$ pc from the cavity center $)$. The size of the cavity is based on the arc structure found by Fukui et al. (2009).

When the shock expands in the cavity, the shock velocity $v_{s}$ is written as a function of 
the SNR age $(t)$ as

$$
v_{s}(t)=\left\{\begin{array}{ll}
v_{i} & \left(t<t_{1}\right) \\
v_{i}\left(t / t_{1}\right)^{-3 / 5} & \left(t_{1}<t<t_{2}\right)
\end{array},\right.
$$

where $v_{i}=v_{i, 9} 10^{9} \mathrm{~cm} \mathrm{~s}^{-1}$ is the initial velocity of the ejecta (Sturner et al. 1997; Yamazaki et al. 2006; Fang \& Zhang 2008). At $t<t_{1}=2.1 \times 10^{2}\left(E_{51} / n_{0}\right)^{1 / 3} v_{i, 9}^{-5 / 3} \mathrm{yr}$, the SNR is in the free expansion phase, at $t>t_{2}=4.0 \times 10^{4} E_{51}^{4 / 17} n_{2}^{-9 / 17} \mathrm{yr}$, it is in the radiative phase, and at $t_{1}<t<t_{2}$, it is in the Sedov phase. Here $n_{0}=100 n_{2} \mathrm{~cm}^{-3}$ and $n_{0}=\mu n_{\text {cav }} \mathrm{cm}^{-3}$, where $n_{\text {cav }}$ is the hydrogen density in the cavity and $\mu=1.4$ is the mean atomic weight of the gas assuming one helium atom for every 10 hydrogen atoms. The initial energy of the ejecta is $E_{\mathrm{SN}}=E_{51} 10^{51} \mathrm{erg}$. The radius of the shock $\left(r_{s}\right)$ can be obtained by integrating equation (3).

The energy spectrum of the accelerated protons is given by

$$
N_{p}(E) \propto E^{-s} \exp \left(-E / E_{\max , \mathrm{p}}\right) .
$$

The index is given by $s=\left(r_{\text {com }}+2\right) /\left(r_{\text {com }}-1\right)$, where $r_{\text {com }}$ is the compression ratio of the shock (Blandford \& Eichler 1987), which is given by the Rankine-Hugoniot relation for $t_{1}<t<t_{2}$. We do not consider non-linear effects, and thus $r_{\text {com }}$ is equal to or smaller than 4. We assume that the maximum energy is determined by the age of the SNR:

$$
E_{\text {max }, \mathrm{p}}=1.6 \times 10^{2} h^{-1}\left(\frac{v_{s}}{10^{8} \mathrm{~cm} \mathrm{~s}^{-1}}\right)^{2}\left(\frac{B_{\mathrm{d}}}{10 \mu \mathrm{G}}\right)\left(\frac{t}{10^{5} \mathrm{yr}}\right) \mathrm{TeV},
$$

where $h(\sim 1)$ is the factor determined by the shock angle and the gyro-factor, and $B_{\mathrm{d}}$ is the downstream magnetic field, which is given by $B_{\mathrm{d}}=r_{\text {com }} B_{\text {cav }}$, where $B_{\text {cav }}$ is the magnetic field in the cavity (Yamazaki et al. 2006). The minimum energy of the protons is given by the rest-mass energy.

In our fiducial model, we assume that $n_{\text {cav }}=4 \mathrm{~cm}^{-3}$, which is the lower end of the typical value in such regions $\left(\sim 5-25 / \mu \mathrm{cm}^{-3}\right.$; Chevalier 1999). There is no information about the temperature and magnetic field in the cavity before the explosion. Thus, we assume that $T_{\text {cav }}=8 \times 10^{5} \mathrm{~K}$ and $B_{\text {cav }}=20 \mu \mathrm{G}$ so that the photon spectrum is consistent with observations; $T_{\text {cav }}$ and $B_{\text {cav }}$ determine $s$ and $E_{\text {max,p }}$, respectively. It is to be noted that the current temperature of the warm gas is $1.4 \times 10^{6}-4.9 \times 10^{7} \mathrm{~K}$ (Fujita et al. 2009). For $v_{i, 9}=E_{51}=1$, the shock radius is $r_{s}=20 \mathrm{pc}$ at $t=1.6 \times 10^{4} \mathrm{yr}$. We define this time as $t_{\text {coll }}$ and the shock collides with the molecular clouds around this time. Since $t_{2}=1.6 \times 10^{4} \mathrm{yr}$, the SNR is at the end of the Sedov phase at $t=t_{\text {coll }}$. Assuming that particle acceleration stops at $t \sim t_{2}$, the protons accelerated at $t \lesssim t_{\text {coll }}\left(\sim t_{2}\right)$ illuminate the molecular clouds. For $t_{1}<t<t_{\text {coll }}$, the compression ratio $r_{\text {com }}$ and the proton cut-off energy $E_{\text {max,p }}$ decrease from 4 to 3.6 and from 151 to $47 \mathrm{TeV}$, respectively. Therefore, the protons that illuminate 
the molecular clouds have a spectrum represented by equation (4) with $2.0 \leq s \leq 2.3$ and $47 \leq E_{\text {max }, \mathrm{p}} \leq 151 \mathrm{TeV}$.

The mass of the molecular clouds around Westerlund 2 is $\sim 2 \times 10^{5} \mathrm{M}_{\odot}$ (Furukawa et al. 2009), although the clouds are very irregular. Referring to the observations, we assume that the proton density of the clouds is $n_{c}=1.0 \times 10^{3} \mathrm{~cm}^{-3}$. In the clouds, we expect that the shock follows the "snowplow" evolution $\left(t>t_{\text {coll }}\right)$. We assume that the shock in the clouds can locally be expressed as a shell centered on $r=0$. In this case, from the momentum conservation, the radius of the shell, $r_{\mathrm{sh}}(t)$, has a relation of

$$
\frac{4 \pi}{3}\left[n_{c}\left(r_{\mathrm{sh}}(t)^{3}-r_{s}\left(t_{\mathrm{coll}}\right)^{3}\right)+n_{\mathrm{cav}} r_{s}\left(t_{\mathrm{coll}}\right)^{3}\right] \dot{r}_{\mathrm{sh}}(t)=\frac{4 \pi}{3} n_{\mathrm{cav}} r_{s}\left(t_{\mathrm{coll}}\right)^{3} v_{s}\left(t_{\mathrm{coll}}\right),
$$

with $r_{\mathrm{sh}}=r_{s}$ at $t=t_{\text {coll }}$, which can be solved numerically for $t>t_{\text {coll }}$. After the collision, it takes $t_{\text {stop }} \sim 1.8 \times 10^{4} \mathrm{yr}$ until the shell velocity decreases to the internal velocity of the clouds $\left(\sim 20 \mathrm{~km} \mathrm{~s}^{-1}\right.$; Furukawa et al. 2009). Since the shock has not been observed apparently, its velocity may have decreased to that level.

Some of the accelerated protons plunge into the molecular clouds and create electrons, positrons, and gamma-ray photons through $p p$-interactions there. The electrons and positrons (both are called secondary electrons) also emit photons through synchrotron, IC, and bremsstrahlung radiation. Figure 2 shows the broad-band spectrum of the molecular clouds at $t \sim t_{\text {coll }}$. We assume that the spectrum of the cosmic-ray protons is represented by equation (4) with $s=2.3$ and $E_{\max , \mathrm{p}}=47 \mathrm{TeV}$. The total energy of the protons illuminating the molecular clouds is $4.5 \times 10^{49} \mathrm{erg}$. Note that the total number of the target protons and the total energy of the illuminating protons are degenerated for the photon spectrum. The magnetic field in the cloud is $B_{c}=60 \mu \mathrm{G}$. The spectrum is calculated based on the radiation models in Fang \& Zhang (2008). For the $p p$-interactions, we used the code provided by Karlsson \& Kamae (2008). In Figure 2, we included the contribution of background cosmic-rays (equation [10] in Gabici et al. 2009), but it is negligibly small. We assumed that the injection of secondary electrons is balanced with the cooling. The model results are compared with observations. The radio flux is considered as an upper limit, because it could only partially include the non-thermal radio flux produced by the energetic electrons (Whiteoak \& Uchida 1997). For the two-band Fermi observations, we assume that the spectral index is between -2.5 and -2 . The data were particularly important to constrain the value of $s$.

The diffusion time of cosmic-rays in a cloud is written as

$$
t_{\mathrm{diff}} \sim \frac{L_{c}^{2}}{6 D(E)}=1.6 \times 10^{4}\left(\frac{\chi}{0.01}\right)^{-1}\left(\frac{L_{c}}{15 \mathrm{pc}}\right)^{2}\left(\frac{E}{10 \mathrm{TeV}}\right)^{-0.5}\left(\frac{B_{c}}{60 \mu \mathrm{G}}\right)^{0.5} \mathrm{yr}
$$


where $L_{c}$ is the size of a molecular cloud. From the condition of $t_{\text {diff }} \gtrsim t_{\text {stop }}$, the reduction factor must be $\chi \lesssim 0.01$. If we ignore the diffusion of the protons in the cloud, the emission originated from the protons will not change for a long time because of the long cooling time of the protons (>105 yr; Gabici et al. 2009).

In Figure 2, the emission from primary electrons is also presented. Their energy spectrum is given by

$$
N_{e}(E) \propto E^{-s} \exp \left(-E / E_{\max , \mathrm{e}}\right) .
$$

The maximum energy is determined by synchrotron cooling:

$$
E_{\text {max }, \mathrm{e}}=14 h^{-1 / 2} v_{s, 8}\left(B_{\mathrm{d}} / 10 \mu \mathrm{G}\right)^{-1 / 2} \mathrm{TeV},
$$

(Yamazaki et al. 2006). The minimum energy of the electrons is given by the rest-mass energy. We assume that the electron-proton ratio is $K_{\mathrm{ep}}=0.01$. If the bremsstrahlung radiation from primary electrons were more than ten times brighter than that in Figure 2 , it might overwhelm the gamma-ray emission from the pion decay. However, the required electron-proton ratio $\left(K_{\mathrm{ep}} \gtrsim 0.1\right)$ would be unrealistically large. Moreover, because of the short cooling time, the radiation of the primary electrons should decrease after particle acceleration stops. Thus, for $t>t_{\text {coll }}\left(\sim t_{2}\right)$, observed flux from the primary electrons should be lower than that in Figure 2 .

\subsection{W 28}

We apply the same model to W 28. A supernova explodes in a low-density cavity (Fig. 1b). The distance to W 28 has been estimated to be $d \sim 2-3 \mathrm{kpc}$ (Goudis 1976; Lozinskava 1981); we assume $d=2.6 \mathrm{kpc}$ in this letter. We assume that $n_{\text {cav }}=8 \mathrm{~cm}^{-3}$, $B_{\text {cav }}=2 \mu \mathrm{G}$, and $T_{\text {cav }}=1 \times 10^{6} \mathrm{~K}$. For $v_{i, 9}=1$ and $E_{51}=0.4$ (Rho \& Borkowski 2002), the shock radius is $r_{s}=11 \mathrm{pc}$ at $t=t_{2}=9.0 \times 10^{3} \mathrm{yr}$. During the Sedov phase, the index $s$ increases from 2.0 to 2.3 , and the maximum proton energy $E_{\text {max,p }}$ decreases from 8.8 to $2.7 \mathrm{TeV}$. At $t \sim t_{2}$, we assume that the shock collides with a surrounding relatively dense region of $n_{h}=70 \mathrm{~cm}^{-3}$ (Fig. 3b) and we define this time as $t=t_{\text {coll }}$. The density $n_{h}$ is based on observations (Long et al. 1991; Reach \& Rho 2000). The detection of 1720 $\mathrm{MHz}$ maser emission from W 28 actually indicates the presence of the shock in the dense gas (Frail et al. 1994; Claussen et al. 1997). After the collision, the evolution of the shock follows equation (6) in which $n_{c}$ is replaced by $n_{h}$. The radius of the shell increases from $r_{\mathrm{sh}}=11$ to $13 \mathrm{pc}$, and the velocity decreases from $v_{s}=500$ to $80 \mathrm{kms}^{-1}$ in $t_{\text {stop }} \sim 1.4 \times 10^{4} \mathrm{yr}$. The radius of $r_{s}=13 \mathrm{pc}$ and the velocity of $80 \mathrm{~km} \mathrm{~s}^{-1}$ are comparable to the observed ones (Bohigas et al. 1983; Rho \& Borkowski 2002). Note that even if particles can be accelerated 
at radiative shocks, it cannot be applied to W 28 at present, because the small velocity $\left(80 \mathrm{~km} \mathrm{~s}^{-1}\right)$ prevents ionization of the preshock gas (Shull \& McKee 1979).

Since the gamma-ray emission comes from clumps around the SNR (Aharonian et al. 2008), we expect that there are dense clumps, which are the targets of cosmic-ray protons (Fig. 1b). Referring to the observations, we assume that the mass and density of a clump is $4 \times 10^{4} M_{\odot}$ and $n_{c}=5.4 \times 10^{2} \mathrm{~cm}^{-3}$, respectively (Aharonian et al. 2008). Figure 3 shows the broad-band spectrum of the molecular cloud at $t \sim t_{\text {coll }}$. The spectrum of the cosmic-ray protons is represented by equation (4) with $s=2.3$ and $E_{\text {max,p }}=2.7 \mathrm{TeV}$. The total energy of the protons that contribute to the illumination of the molecular clouds is $2 \times 10^{49} \mathrm{erg}$. The magnetic field in the cloud is $B_{\mathrm{c}}=60 \mu \mathrm{G}$. The radio flux is considered as an upper limit, because it includes radio emission outside of the region where $\mathrm{TeV}$ gammaray has been detected (Dubner et al. 2000). Since the XMM-Newton upper-limit is for part of the $\mathrm{TeV}$ gamma-ray region, the actual upper-limit may be larger (Nakamura et al. 2009, in preparation). As is the case for Westerlund 2, while the photon flux from primary electrons decreases as the time advances, that originated from protons does not. The condition $t_{\text {diff }} \gtrsim t_{\text {stop }}$ gives $\chi \lesssim 0.01$. Recently, Fermi found GeV gamma-rays in the molecular clouds where TeV gamma-ray has been detected (Abdo et al. 2009), which indicates that the energy dependence of $D(E)$ is not affecting the proton spectrum and is consistent with our assumption that the protons were accelerated near the molecular clouds.

\section{Summary and Discussion}

In this letter, we constructed a model of gamma-ray emission from molecular clouds surrounding an SNR. We considered old SNRs because gamma-rays mainly come through $p p$-interactions and pion decay, and those from primary electrons can be ignored. In fact, the synchrotron cooling time of electrons with $E \sim 1 \mathrm{TeV}$ is only $\sim 4 \times 10^{3}$ yr for $B \sim 60 \mu \mathrm{G}$ (Gabici et al. 2009), and thus they cool quickly after their acceleration finishes. We assumed that the SNR shock travels in a low-density cavity, and we indicated that the diffusion coefficient of cosmic-ray particles around the SNR needs to be much smaller than that away from it (less than $\sim 1 \%$ ), The predicted broad-band spectra for Westerlund 2 and W 28 are consistent with observations (Figs. 2 and 3). The proton spectra can be fitted with a power law with indices $\sim 2.3$, which is the value at cosmic-ray sources expected in cosmic-ray

propagation models for the Galaxy (Strong et al. 2007). The indices suggest that particles were accelerated near the molecular clouds even after the SNR became old and large for these objects. The soft spectra may be because the particles were accelerated at the end of the Sedov phase. While the low Mach number of the shock makes the spectra soft in 
our model, neutral hydrogens in the cavity may also do that (Ohira, Terasawa, \& Takahara 2009). On the other hand, for another SNR IC 443, the broad-band photon spectrum indicates that the index is 2 (Zhang \& Fang 2008). For this object, protons accelerated at an earlier stage of the Sedov phase (when the Mach number of the shock is large) may be illuminating molecular clouds. Moreover, if molecular clouds are distributed with various sizes and distances from the SNR, the gamma-ray spectrum may be the superposition of the spectrum of each cloud and may reflect the energy-dependence of particle diffusion (equation [2; see Aharonian \& Atovan 1996; Torres et al. 2008).

We are grateful to Y. Fukui, R. Nakamura, A. Bamba, R. Yamazaki, and K. Kohri for useful discussion. This work was supported by KAKENHI (Y. F.: 20540269; Y. O.: 20.1697; F. T.; 20540231).

\section{REFERENCES}

Abdo, A. A., et al. 2009, ApJS, 183, 46

Aharonian, F., et al. 2007, A\&A, 467, 1075

Aharonian, F., et al. 2008, A\&A, 481, 401

Aharonian, F. A., \& Atoyan, A. M. 1996, A\&A, 309, 917

Atoyan, A. M., Aharonian, F. A., Völk, H. J. 1995, Phys. Rev. D, 52, 3265

Baring, M. G., Ellison, D. C., Reynolds, S. P., Grenier, I. A., \& Goret, P. 1999, ApJ, 513, 311

Berezhko, E. G., Völk, H. J. 2000, ApJ, 540, 923

Blandford, R., \& Eichler, D. 1987, Phys. Rep., 154, 1

Bohigas, J., Ruiz, M. T., Carrasco, L., Salas, L., \& Herrera, M. A. 1983, Revista Mexicana de Astronomia and Astrofisica, 8, 155

Chevalier, R. A. 1999, ApJ, 511, 798

Claussen, M. J., Frail, D. A., Goss, W. M., \& Gaume, R. A. 1997, ApJ, 489, 143

Drury, L. O., Aharonian, F. A., \& Völk, H. J. 1994, A\&A, 287, 959

Dubner, G. M., Velázquez, P. F., Goss, W. M., \& Holdaway, M. A. 2000, AJ, 120, 1933 
Fang, J., \& Zhang, L. 2008, MNRAS, 384, 1119

Fatuzzo, M., Adams, F. C., \& Melia, F. 2006, ApJ, 653, L49

Fatuzzo, M., \& Melia, F. 2005, ApJ, 630, 321

Frail, D. A., Goss, W. M., \& Slysh, V. I. 1994, ApJ, 424, L111

Fujita, Y., Hayashida, K., Takahashi, H., \& Takahara, F. 2009, arXiv:0907.4750, PASJ, in press

Fukui, Y., et al. 2009, PASJ, 61, L23

Furukawa, N., Dawson, J. R., Ohama, A., Kawamura, A., Mizuno, N., Onishi, T., \& Fukui, Y. 2009, ApJ, 696, L115

Gabici, S., Aharonian, F. A., \& Casanova, S. 2009, MNRAS, 396, 1629

Gaisser, T. K., Protheroe, R. J., \& Stanev, T. 1998, ApJ, 492, 219

Goudis, C. 1976, Ap\&SS, 40, 91

Karlsson, N., \& Kamae, T. 2008, ApJ, 674, 278

Koyama, K., Petre, R., Gotthelf, E. V., Hwang, U., Matsuura, M., Ozaki, M., \& Holt, S. S. 1995, Nature, 378, 255

Long, K. S., Blair, W. P., Matsui, Y., \& White, R. L. 1991, ApJ, 373, 567

Lozinskaya, T. A. 1981, Soviet Astronomy Letters, 7, 17

Naito, T., \& Takahara, F. 1994, Journal of Physics G Nuclear Physics, 20, 477

Ohira, Y., Terasawa, T., \& Takahara, F. 2009, ApJ, 703, L59

Reach, W. T., \& Rho, J. 2000, ApJ, 544, 843

Rho, J., \& Borkowski, K. J. 2002, ApJ, 575, 201

Shull, J. M., \& McKee, C. F. 1979, ApJ, 227, 131

Strong, A. W., Moskalenko, I. V., \& Ptuskin, V. S. 2007, Annual Review of Nuclear and Particle Science, 57, 285

Sturner, S. J., Skibo, J. G., Dermer, C. D., \& Mattox, J. R. 1997, ApJ, 490, 619 
Torres, D. F., Rodriguez Marrero, A. Y., \& de Cea Del Pozo, E. 2008, MNRAS, 387, L59

Wentzel, D. G. 1974, ARA\&A, 12, 71

Whiteoak, J. B. Z., \& Uchida, K. I. 1997, A\&A, 317, 563

Wootten, A. 1981, ApJ, 245, 105

Yamazaki, R., Kohri, K., Bamba, A., Yoshida, T., Tsuribe, T., \& Takahara, F. 2006, MNRAS, 371, 1975

Zhang, L., \& Fang, J. 2008, ApJ, 675, L21 


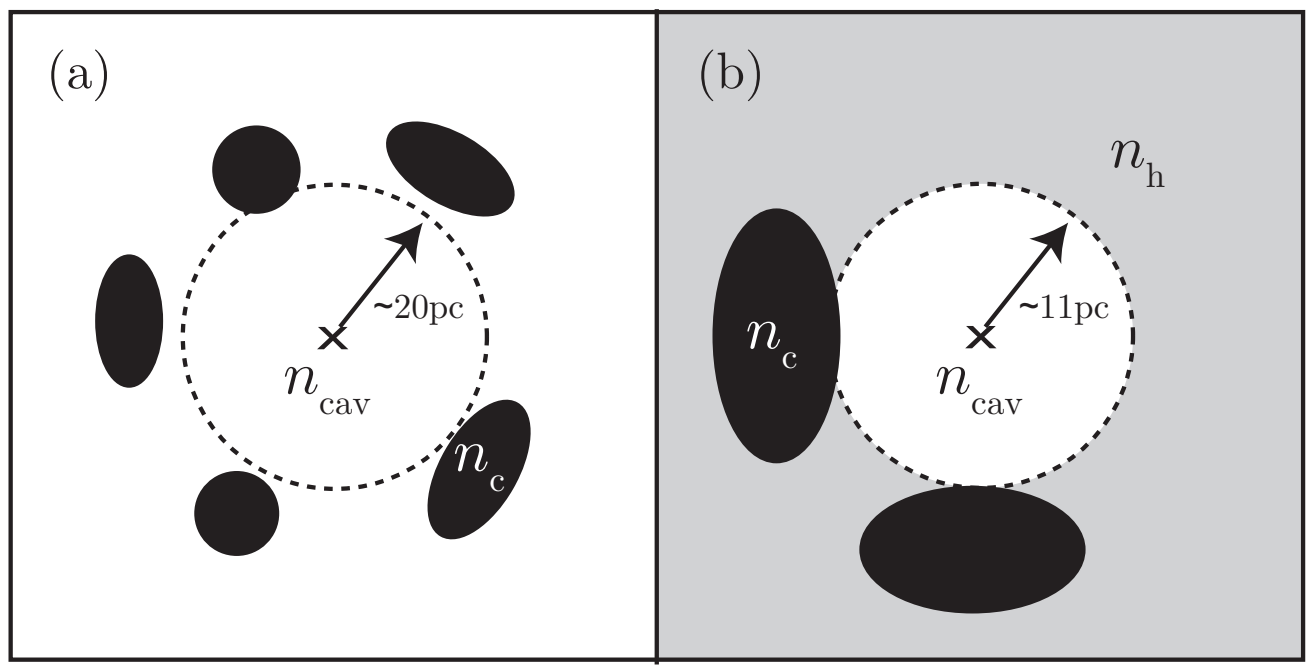

Fig. 1.- Configuration of the cavity and clouds. (a) Westerlund 2 and (b) W 28. 


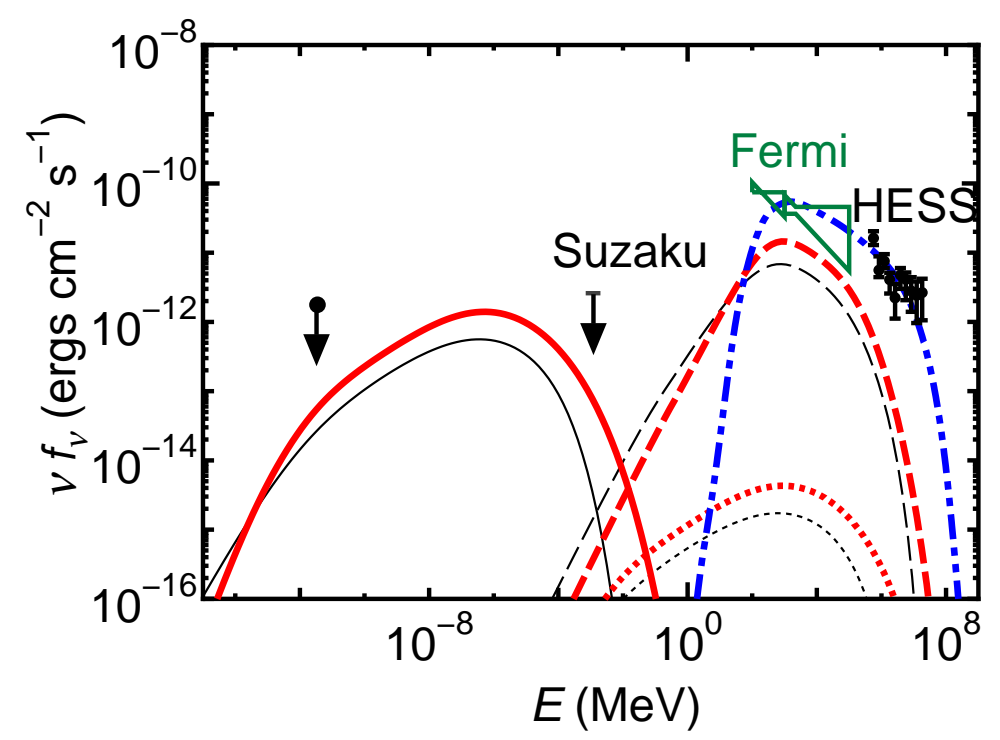

Fig. 2.- Comparison of the model results with radio (Whiteoak \& Uchida 1997), Suzaku (Fuiita et al. 2009), Fermi (Abdo et al. 2009), and H.E.S.S. observations (Aharonian et al. 2007) for Westerlund 2. The synchrotron radiation (solid line), bremsstrahlung (dashed line), and IC scattering (dotted line) are of the primary electrons (thin lines) and the secondary electrons (thick lines). The $\pi^{0}$ decay gamma-rays are shown by the two-dot-dashed line.

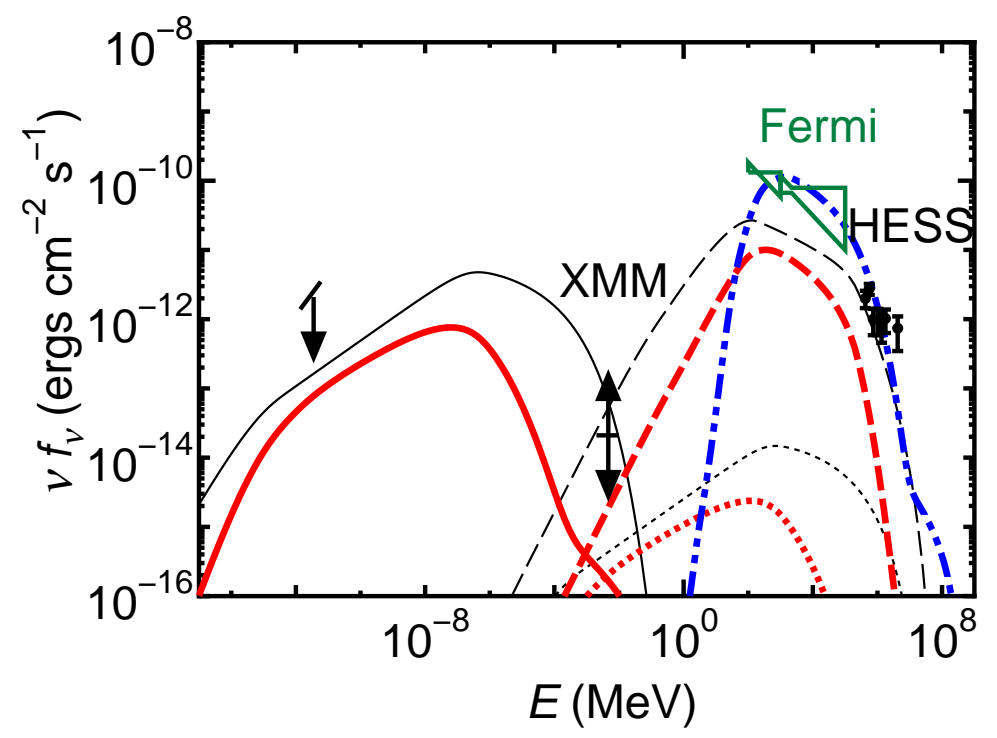

Fig. 3.- Comparison of the model results with radio (Dubner et al. 2000), XMM-Newton (Nakamura et al. 2009, in preparation), and Fermi observations (Abdo et al. 2009) for W 28. H.E.S.S. observations are for HESS J1801-233 (Aharonian et al. 2008). The lines are the same as those in Fig. 2. 